\title{
Dynamics of intraday serial correlation in the Italian futures market*
}

\author{
Simone Bianco \\ Center for Nonlinear Science, University of North Texas \\ P.O. Box 31 1427, Denton, Texas, 76201-1427 \\ e-mail: sbianco@unt.edu \\ Roberto Renò \\ Dipartimento di Economia Politica, Università di Siena \\ Piazza S.Francesco 7, 53100, Siena \\ e-mail: reno@unisi.it
}

22nd March 2005

\begin{abstract}
We study the serial correlation of high-frequency intraday returns on the Italian stock index futures (FIB30) in the period 2000-2002. We find that intraday autocorrelation is mostly negative for time scales lower than 20 minutes, mainly due to the bid-ask bounce effect. While this supports the efficiency of the Italian futures market, we also provide evidence that intraday serial correlation becomes positive in high volatility regimes. Moreover we find that it is mainly unexpected volatility to make serial correlation rise, and not its predictable part. Our results are supportive of the Chan (1993) model.
\end{abstract}

${ }^{*}$ We wish to acknowledge Borsa Italiana SPA, and in particular Concetta Ricciardi and Ada De Roma, for providing the excellent data set. We also acknowledge Rosario Rizza, Maria Pasquale and Carmelo Genovese for research assistance, as well as the Department of Physics of the University of Pisa. Giampiero M. Gallo, Tarcisio del Prete and Fulvio Corsi provided useful comments. We finally acknowledge the participants at the II Workshop on high-frequency data held in Perugia, 2004. All errors and omissions are our own. 


\section{Introduction and motivation}

The aim of this paper is to study the serial correlation of the Italian stock index futures. The study of serial correlation of asset returns is particularly important in financial economics, since it can reveal basic features of the trading process. The Efficient Market Hypothesis in its weakest form implies that asset returns should be serially uncorrelated, but there is pervasive evidence of serial autocorrelation in stock index returns (Lo and MacKinlay, 1988; Poterba and Summers, 1988) and stock portfolios (Conrad and Kaul, 1988; Mech, 1993), mixed evidence on stocks (Lo and MacKinlay, 1990b; Conrad and Kaul, 1989; Kim et al., 1991) and international assets (Patro and $\mathrm{Wu}, 2004)$, mainly depending on volumes and size (Llorente et al., 2002), while stock index futures display no autocorrelation, see Ahn et al. (2002) and Pan et al. (1997) for currency futures. There are many theoretical models and explanations, on one side to reconcile the presence of serial correlation with a rational framework, e.g. non-synchronous trading (Lo and MacKinlay, 1990a) or institutional factors (Boudoukh et al., 1994), and on the other side to advocate for bounded rationality in financial markets, e.g. Cutler et al. (1991); Jegadeesh and Titman (1993); Badrinath et al. (1995).

In our study, starting from the observation that stock index futures are not serially correlated at the daily level, we go in the high-frequency regime to study correlation and its link with some of the most important quantities of the financial market, that is volatility and trading volume. We test for the presence of serial correlation using the Variance Ratio test, after controlling for the peculiarities which are typical of high-frequency transaction data.

While high-frequency transactions are nowadays a common tool in financial econometrics (Goodhart and O'Hara, 1997), the study of serial correlation with high frequency data is, to our knowledge, very limited. Examples are Low and Muthuswamy (1996) on exchange rates and Thomas and Patnaik (2003) on the Indian market; in this last paper serial correlation is linked with liquidity. Typically, high-frequency data are difficult to deal with because of their irregular structure and microstructure effects. Moreover, they are characterized by strong intraday seasonalities and pronounced heteroskedasticity, see e.g. Bollerslev and Domowitz (1993); Dacorogna et al. (1993); Andersen and Bollerslev (1997) among others. For this reason, Andersen et al. (2001) strongly criticize the adoption of Variance Ratio with high frequency data, as in Ito et al. (1998), for testing for changes 
in intraday volatility patterns. Then, we first check via Monte Carlo simulations whether the small sample distribution of the VR statistics matches the asymptotic distribution.

We then compute daily VR statistics on intraday transactions of the FIB30. Our findings show that, as expected, intraday serial correlation is mostly negative for time scales lower than 20 minutes, mostly due to the bid-ask spread. We then use daily measure of VRs as a dynamic quantity that can be related to other market variables. We exploit the availability of high-frequency data to compute daily measures of integrated volatility, and as a consequence we can directly link the intraday serial correlation (as measured by the variance ratio) to daily volatility. Using this technique, we find that intraday serial correlation becomes significantly positive when volatility is high. Moreover, intraday serial correlation turns out to be positively linked to trading volume. We also show that serial correlation is more linked to the unexpected part of volatility than to volatility itself, which is highly predictable.

This paper is structured as follows. Section 2 describes the variance ratio statistics, and discusses the implementation on high-frequency data. Preliminary data analysis is worked out in Section 3. We then study the dynamics of serial correlations in Section 4 . Section 5 concludes.

\section{The Variance Ratio test and its implementa- tion on intraday data}

We decide to adopt a popular test in this kind of analysis, the Variance Ratio test $(V R)$. This test has been extensively used for daily asset prices, see e.g. Lo and MacKinlay (1988, 1989). The Variance Ratio test has not been used too much for high frequency data. One example close to our study is the work of Thomas and Patnaik (2003). In this Section we analyze briefly the basic properties of this test.

In our description, we follow closely Lo and MacKinlay (1988). Suppose we have a time series $P_{k}, k=1, \ldots, N$ of asset prices or logarithmic asset prices. Define the first differences time series $r_{k}=P_{k}-P_{k-1}$, then define:

$$
\operatorname{VR}(q)=\frac{\operatorname{Var}[r(q)]}{\operatorname{Var}\left[r_{k}\right]}=1+\sum_{k=1}^{q}\left(1-\frac{k}{q}\right) \rho_{k},
$$


where

$$
r(q)=\sum_{k=1}^{q+1} r_{k}
$$

represents the $q$-period return and $\rho_{k}$ is the standard autocorrelation function at lag $k$. Equation (1) highlights the link between variance ratio and the autocorrelation function. Under the weak form efficient market hypothesis the dynamics of returns has to be a random walk and then, immediately, $\rho_{k}=0, \forall k$. That implies $\mathbb{E}[V R(q)]=1$.

We implement the variance ratio test according to the heteroskedasticconsistent estimator (Lo and MacKinlay, 1988) with overlapping observations (Richardson and Smith, 1993). Suppose to have a set of $n q+1$ observations, where $q$ is an integer greater than 1 . We then define:

$$
\begin{array}{r}
\hat{\mu} \equiv \frac{1}{n q} \sum_{k=1}^{n q}\left(P_{k}-P_{k-1}\right)=\frac{1}{n q}\left(P_{n q}-P_{0}\right) \\
\tilde{\sigma}_{a}^{2} \equiv \frac{1}{n q-1} \sum_{k=1}^{n q}\left(P_{k}-P_{k-1}-\hat{\mu}\right)^{2} \\
\tilde{\sigma}_{c}^{2} \equiv \frac{1}{m} \sum_{k=q}^{n q}\left(P_{k}-P_{k-q}-q \hat{\mu}\right)^{2}
\end{array}
$$

where

$$
m=q(n q-q+1)\left(1-\frac{q}{n q}\right) .
$$

We define the variance ratio as follows:

$$
\widehat{V R}(q)=\frac{\hat{\sigma}_{c}^{2}}{\hat{\sigma}_{a}^{2}} .
$$

Using overlapping observations leads to an improvement in the power of the test, estimated around 22\% (Richardson and Smith, 1993).

Under the null hypothesis of random walk, the asymptotic distribution of the statistics (7) is the following. Define:

$$
\begin{array}{r}
\hat{\delta}_{k}=\frac{n q \sum_{j=k+1}^{n q}\left(P_{j}-P_{j-1}-\hat{\mu}\right)^{2}\left(P_{j-k}-P_{j-k-1}-\hat{\mu}\right)^{2}}{\left[\sum_{j=1}^{n q}\left(P_{j}-P_{j-1}-\hat{\mu}\right)^{2}\right]^{2}} \\
\hat{\theta}(q)=4 \sum_{k=1}^{q-1}\left(1-\frac{k}{q}\right)^{2} \hat{\delta}_{k} .
\end{array}
$$


Then we have:

$$
\sqrt{n q}(\widehat{V R}(q)-1) \sim N(0, \hat{\theta}),
$$

The variance ratio test implemented here allows for heteroskedasticity, does not require the assumption of normality and in small samples it is more powerful than other tests, like the Ljung-Box statistics or the Dickey-Fuller unit root test, see Lo and MacKinlay (1989); Faust (1992); Cecchetti and Sang Lam (1994).

Dealing with high frequency data can present some potential difficulties. For example, Andersen et al. (2001) show that, given the high persistence of intraday financial prices, the small-sample test is distorted, thus asymptotic inference can be misleading. However, their test regards the equality of variances in two subsequent time windows, while we want to test the reliability of VRs in a given time window, in order to quantify serial correlation. To show that the small-sample performance of the VR test for our purposes is reliable, we proceed as follows. We first estimate a $\operatorname{GARCH}(1,1)$ model on daily returns: ${ }^{1}$

$$
\begin{aligned}
& r_{t}=\sqrt{h_{t}} \cdot \varepsilon_{t}, \\
& h_{t}=\omega+\alpha \cdot r_{t-1}^{2}+\beta \cdot h_{t-1} .
\end{aligned}
$$

with $\varepsilon_{t} \sim \mathcal{N}(0,1)$. GARCH modeling provides a very good approximation of the heteroskedasticity in financial data, see e.g. Bollerslev et al. (1994). Maximum-likelihood estimates of the model are $\hat{\omega}=0.7281 \cdot 10^{-5}, \hat{\alpha}=0.1339, \hat{\beta}=$ 0.8474. For comparison with the analysis in the subsequent Sections, we then convert the parameters to a 1-minute GARCH model, according to the Drost and Nijman (1993) temporal aggregation formulas ${ }^{2}$, that is we obtain the value of the parameters under which the GARCH model (11) still holds if the time unit is one minute instead of one day. Denoting the new parameters with primes, we obtain $\hat{\omega}^{\prime}=1.594 \cdot 10^{-8}, \hat{\alpha}^{\prime}=0.00733, \hat{\beta}^{\prime}=0.9926$. We then simulate the 1-minute GARCH model; daily time series correspond to 495 1-minute observations. On these simulated high-frequency time series,

\footnotetext{
${ }^{1}$ We actually use daily returns on the stock index, namely the MIB30, for the same time span which will be studied in the next Section, namely January 2000-December 2002 (751 observations).

${ }^{2}$ It has been shown that temporal aggregation of GARCH models does not hold empirically on high frequency data (Andersen et al., 1999; Barucci and Renò, 2002a). However, as noted by Andersen et al. (2001), using the Drost and Nijman (1993) aggregation formulas provides a conservative estimate of the distortions typically encountered in high-frequency data.
} 
we compute the variance ratios and standard errors according to the above expressions. If the small-sample distribution is reliable, then the quantity $\sqrt{n q}(\widehat{V R}(q)-1) / \sqrt{\hat{\theta}(q)}$ should be distributed as a $\mathcal{N}(0,1)$. Figure 1 shows the result for different values of $q$. For simulated time series akin to the real ones, with heteroskedasticity modeled according to a GARCH specification, the small sample estimator performs fairly well for $q=1$ and quite well till $q \simeq 20$. For larger $q$, the small sample distribution is distorted with respect to the asymptotic one.

Our results are in line with those of Deo and Richardson (2003), who study the small sample properties of Variance Ratios. They show that reliability of the asymptotic approximation is worse when $q$ increases, but the approximation works fine with small $q$. More precisely, they show that the VR test loses its power at the $95 \%$ of c.l. for values of $q / n \sim 1 / 6$. We remind that in our case $n=495$, so that $q$ should be less than 80 . We find a lower value $q \simeq 20$ because the heteroskedasticity of intraday data is more pronounced than that of daily data. Then we conclude that for $q \leq 20$ the small sample distribution of the $V R$ statistics is approximatively Normal. The statistics would get distorted for larger $q$. We then study $q=1,10,20$, ranging from one minute to twenty minutes. For these values of $q$, we can then safely use the VR test with high-frequency data in our context.

\section{Preliminary analysis}

Our goal is to study the presence of serial correlation patterns in time series of returns of the Italian stock index futures, named FIB30. While being by far the most liquid traded asset in the Italian stock exchange, the FIB30 is still not very liquid when compared to other marketplaces ${ }^{3}$. The market for FIB30 ${ }^{4}$ started from 28 November 1994. It is a price-driven electronic market, in which market makers are supposed to guarantee the liquidity of the market. An advantage of using the futures is that it is a traded asset. Thus we avoid the problem of non-synchronous trading, which is itself a source of serial correlation. Moreover the stock index futures is always more liquid than the portfolio which constitutes the index, and indeed it is

\footnotetext{
${ }^{3}$ The Italian stock market accounts for nearly $2 \%$ of worldwide stock market capitalization.

${ }^{4}$ From 22 March 2004 this contract has been replaced by the S\&P MIB Futures, since the MIB30 index has been replaced by the S\&P MIB
} 

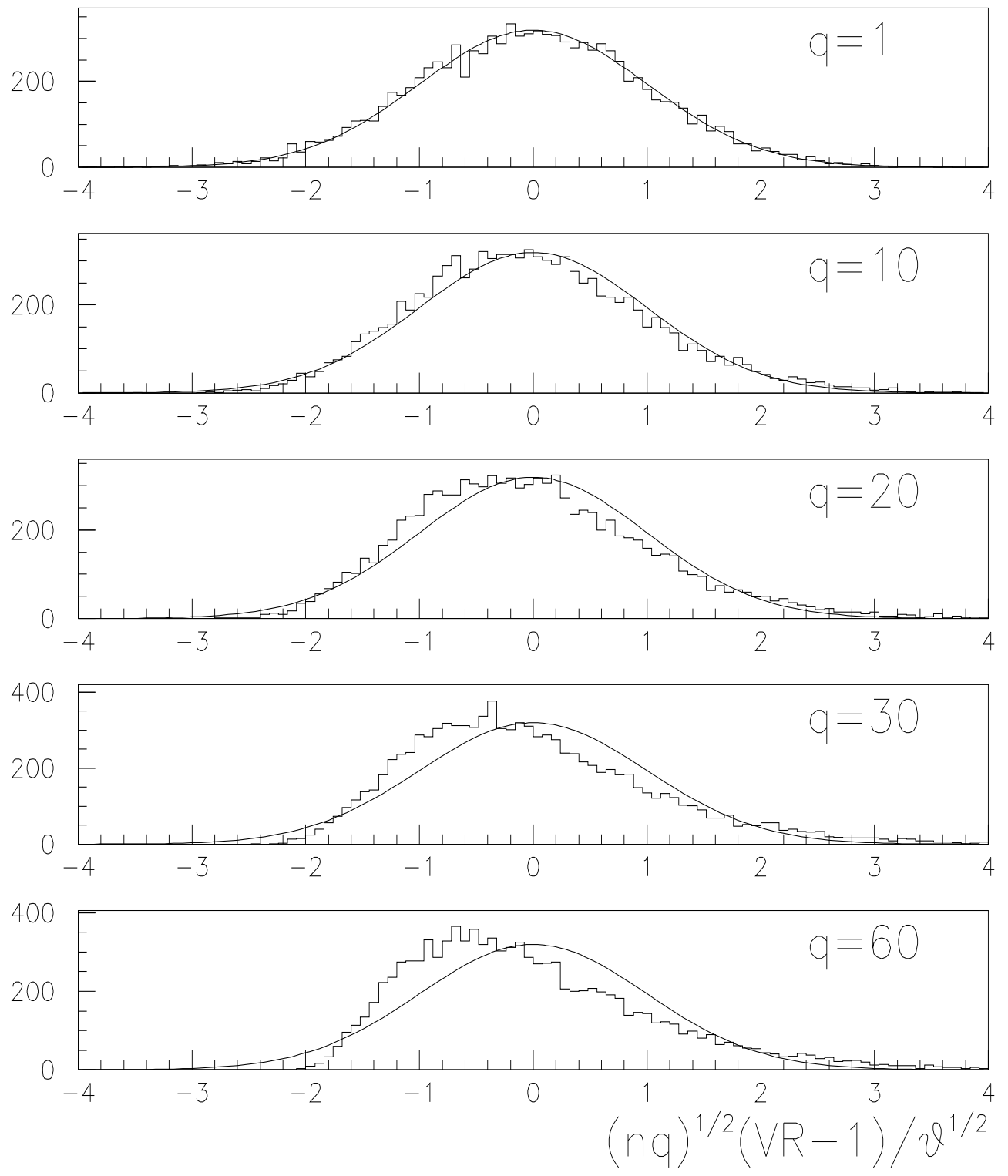

Figure 1: Shows the small sample and asymptotic distributions of VR statistics for different values of $q$. The histograms are the distributions of $\sqrt{n q}(\widehat{V R}(q)-1) / \sqrt{\hat{\theta}(q)}$ on 10,000 simulations of a GARCH $(1,1)$ process of one day of one-minute returns. The solid curves are the asymptotic $\mathcal{N}(0,1)$ distributions. The small sample distribution is very close to the asymptotic one for $q \leq 20$, then it becomes distorted. 
found to lead the stock market (Chan, 1992).

Our data set consists of three years of data, ranging from January 2000 to December 2002, for a total of 751 trading days. We have all the transactions, but we use only those of the next-to-expiration contract, with the FIB30 expiring quarterly. This choice is motivated by the fact that nextto-expiration contract id the most liquid, while the market for the other expirations contract is very thin. We consider only transactions from 9.15 to 17.30 , Italian time. In total, we have $8,657,949$ transactions (on average, 11,528 per day and an average duration between adjacent trades of 2.61 seconds).

Our aim is to compute the variance ratio statistics on logarithmic futures prices every day, then to use it as a dynamical variable. The data set is made up of tick-by-tick transactions. This fact implies that our data are not evenly spaced. In order to implement the VR test according to the expressions in the previous Section, we need an interpolation procedure on a fixed time grid, made of intervals of duration $\Delta t$.

Denoting by $[0, T]$ the time window under study, with $T=1$ trading day, that is 495 minutes, we construct a grid of $T / \Delta t+1$ points, and at every point in time, the price is defined as the price of last transaction before that point ${ }^{5}$. Selecting the length $\Delta t$ of the intervals is conditioned by the following trade-off. If the interval is too narrow, we do not get rid of microstructure effect, such as the bid-ask bouncing, which would induce a spurious negative serial correlation. On the other hand, if the interval is too large, we lose statistics.

In order to assess the proper length of the interval $\Delta t$ for our study, we look at the daily variance ${ }^{6}$ computed as the sum of squared returns, defined as:

$$
\sigma_{d}^{2}(\Delta t)=\sum_{k=0}^{\frac{T}{\Delta t}}[p(k \Delta t+1)-p(k \Delta t)]^{2},
$$

where $p(t)$ is the logarithmic futures price at time $t$, as a function of the interval width $\Delta t$. If the price driving process is a stochastic differential equation with no drift, then the expected value of $\sigma_{d}^{2}(\Delta t)$ equals the integrated variance over the time window $[0, T]$, for every value of $\Delta t$, see BarndorffNielsen and Shephard (2002). If there are microstructure effects, and the

\footnotetext{
${ }^{5}$ Different choices, like linear interpolation, would induce spurious autocorrelation in the data, see Barucci and Renò (2002b); Corsi et al. (2001); Kanatani (2004).

${ }^{6} \mathrm{By}$ defining the variance in this way, we are implicitly assuming that the mean of returns is zero, which is common when dealing with intraday data.
} 


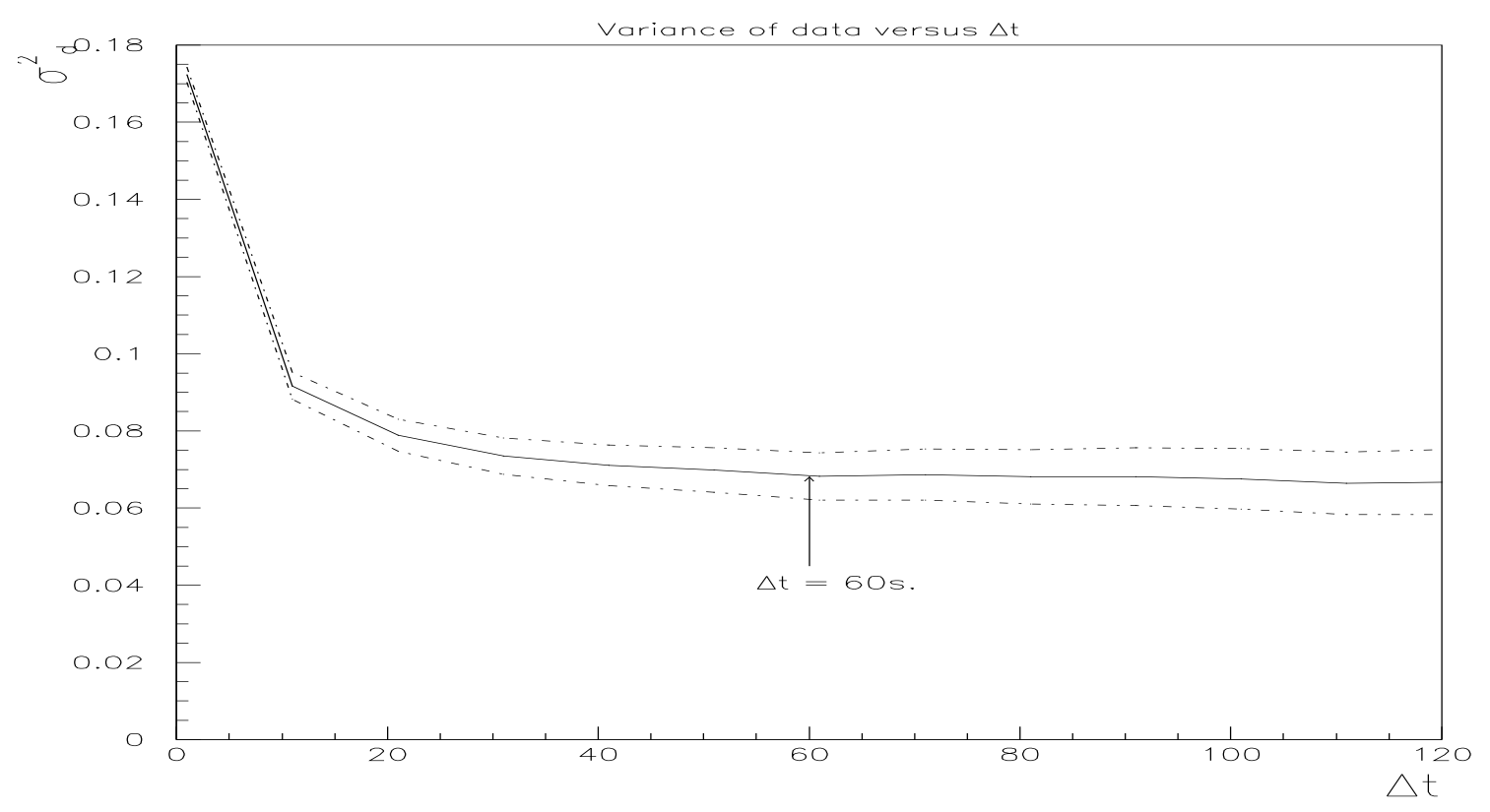

Figure 2: Average daily variance $\sigma_{d}^{2}$ of the data set as a function of the interval $\Delta t$, see equation (12). Dashed lines indicate $95 \%$ confidence bands.

continuous diffusion is no longer a good approximation, this scaling property does not hold any more. Preliminarely, we then analyze the variance of the data versus the aggregation period $\Delta t$, that is, in other word, the length of the sampling interval. Figure 2 shows the variance $\sigma_{d}^{2}(\Delta t)$ averaged over the whole sample. As we can see, the variance increases when $\Delta t$ is small. This effect is well known, see Andersen et al. (2000); Barucci and Renò (2002a), and it can be imputed to the presence of negative serial correlation due to microstructure noise (i.e. bid-ask spread). This problem is the same encountered when choosing the time interval when computing realized volatility, see e.g. Andersen et al. (2003). When $\Delta t$ increases, the value for the variance converges to a constant. After looking at Figure 2, we conclude that a reasonable choice to eliminate microstructure effects is $\Delta t=60$ seconds $^{7}$. Moreover, at $\Delta t=60$ seconds we also eliminate the problem of non-trading, that is the absence of observations in the intervals, which would induce spurious autocorrelation. Indeed, the percentage of empty one-minute intervals in our sample is just $0.9 \%$.

Following the above considerations, each day in our data sample we construct a time series of 495 1-minute returns. On these time series, every

\footnotetext{
${ }^{7}$ We also repeated the analysis with $\Delta t=30$ seconds, with qualitatively similar results, which are available upon request.
} 


\begin{tabular}{lllllll}
\hline$q$ & $90 \%^{+}$ & $90 \%^{-}$ & $95 \%^{+}$ & $95 \%^{-}$ & $99 \%^{+}$ & $99 \%^{-}$ \\
\hline 1 & 0.096 & 0.222 & 0.061 & 0.122 & 0.021 & 0.027 \\
10 & 0.071 & 0.195 & 0.043 & 0.093 & 0.013 & 0.012 \\
20 & 0.055 & 0.172 & 0.031 & 0.063 & 0.004 & 0.003 \\
\hline
\end{tabular}

Table 1: Percentages of VRs significantly greater than 1 (in the columns labeled with ${ }^{+}$) and smaller than 1 (in the columns labeled with ${ }^{-}$), for different significance levels (90, 95, 99\%).

day, we compute the variance ratio using the overlapping statistics (7) and the standard errors using the heteroskedastic consistent estimator (10), for different values $q$. Then, for every value of $q$, we get a time series of daily variance ratios with 751 observations.

If the market is efficient in its weak form (Fama, 1970), then we should get variance ratios compatible with the null hypothesis for every value of $q$. The choice of the maximal value of $q$ that can be considered is limited by the consideration made in the above Section.

Table 1 reports the percentage of VRs statistically significant, both positive and negative. The Table shows that, for small $q$, we find systematic violations of the null, pointing toward negative serial correlation. For example, for $q=1$, we expect around $10 \%$ of violations of the $90 \%$ confidence limit, on the negative tail, but we find $22.2 \%$ of violations. This trend is confirmed till $q \simeq 20$, corresponding to 20 minutes. The positive violations are consistent with the null.

Can we ascribe the significant negative correlation to the bid-ask bounce effect? One way to answer this question is to quantify the implicit bid-ask spread using the negative auto-covariance of the data, as proposed in Roll $(1984)^{8}$. The daily auto-covariance of one-minute high-frequency data is, in average, slightly positive, being equal to $0.105 \cdot 10^{-8}$, with a standard deviation of $7.56 \cdot 10^{-8}$. Thus, since the index ranges around 30,000 points, this would imply a spread of 15 index points ${ }^{9}$, which is a very reasonable bid-ask spread for the Italian futures market (the minimum tick is 5 index points). To be more precise, we compute the exact implied spread for those days in which the auto-covariance of one-minute returns is negative, and we plot the distribution of these spreads in Figure 3. As we can see from the figure,

\footnotetext{
${ }^{8}$ The exact formula is that the relative spread is equal to $2 \sqrt{-C o v}$, where the $C o v$ is the first-order auto-covariance of one-minute returns.

${ }^{9}$ We used Roll's formula plugging in the negative standard deviation.
} 


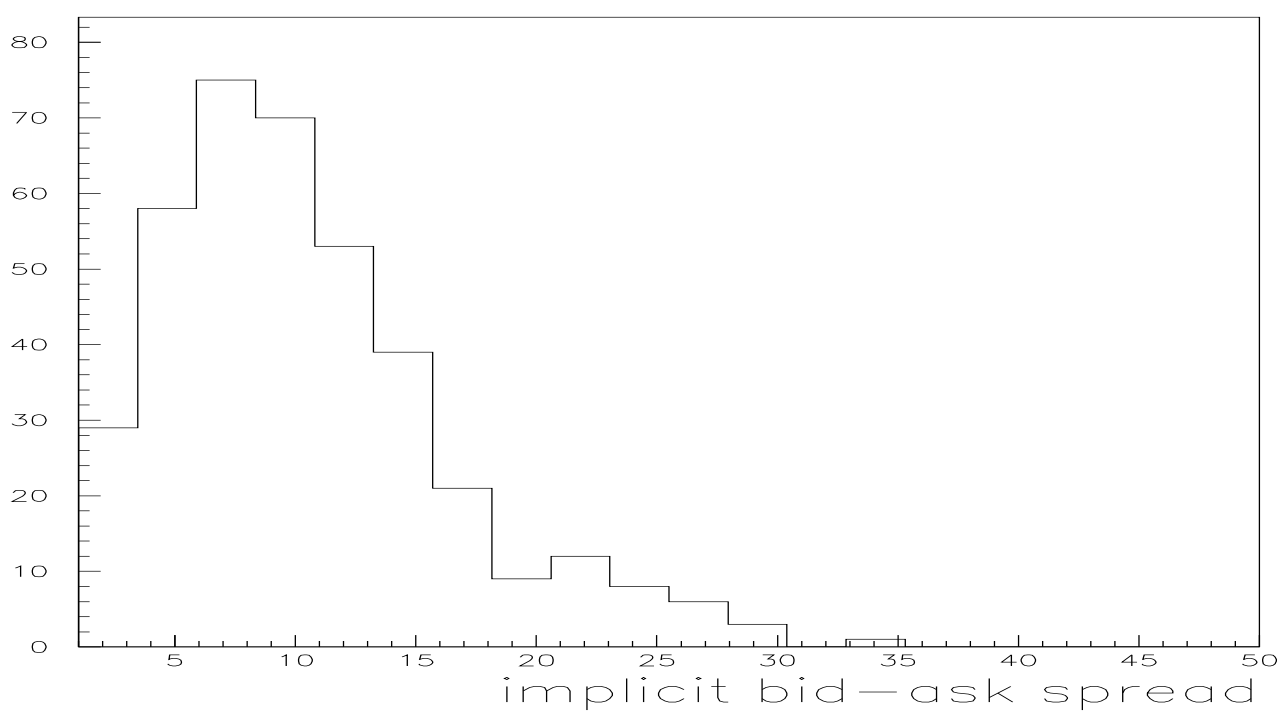

Figure 3: Distribution of implicit bid-ask spreads in the Italian stock index futures according to Roll (1984) measure, which uses the auto-covariance of the data.

when the auto-covariance is negative, the implicit bid-ask spread is always around $5-30$ index points, which is a very reasonable value. Then, the observed negative auto-correlation is completely explained by the bid-ask bounce effect, and we can rule out alternative explanations, like positive feedback trading (Sentana and Wadhwani, 1992). This result supports the efficiency of the Italian futures market.

In order to quantify the daily serial correlation, we use the standardized variance ratio, defined as:

$$
\widetilde{V R}(q)=\sqrt{n q} \frac{\widehat{V R}(q)-1}{\sqrt{\hat{\theta}(q)}}
$$

i.e. the $V R(q)$ normalized with its standard deviation, in the hypothesis of heteroskedasticity and considering overlapping observations. By standardizing, we reduce the impact of large observations estimated with low precision. Figure 4 shows the time series of $\widetilde{V R}(1)$. As we can see, the trend is consistent with Table 1 . There is evidence of serial correlation patterns, mainly negative. By defining a precise quantitative object related to serial correlation, we are able to study its dynamic properties.

The results in this Section can be summarized as follows: 


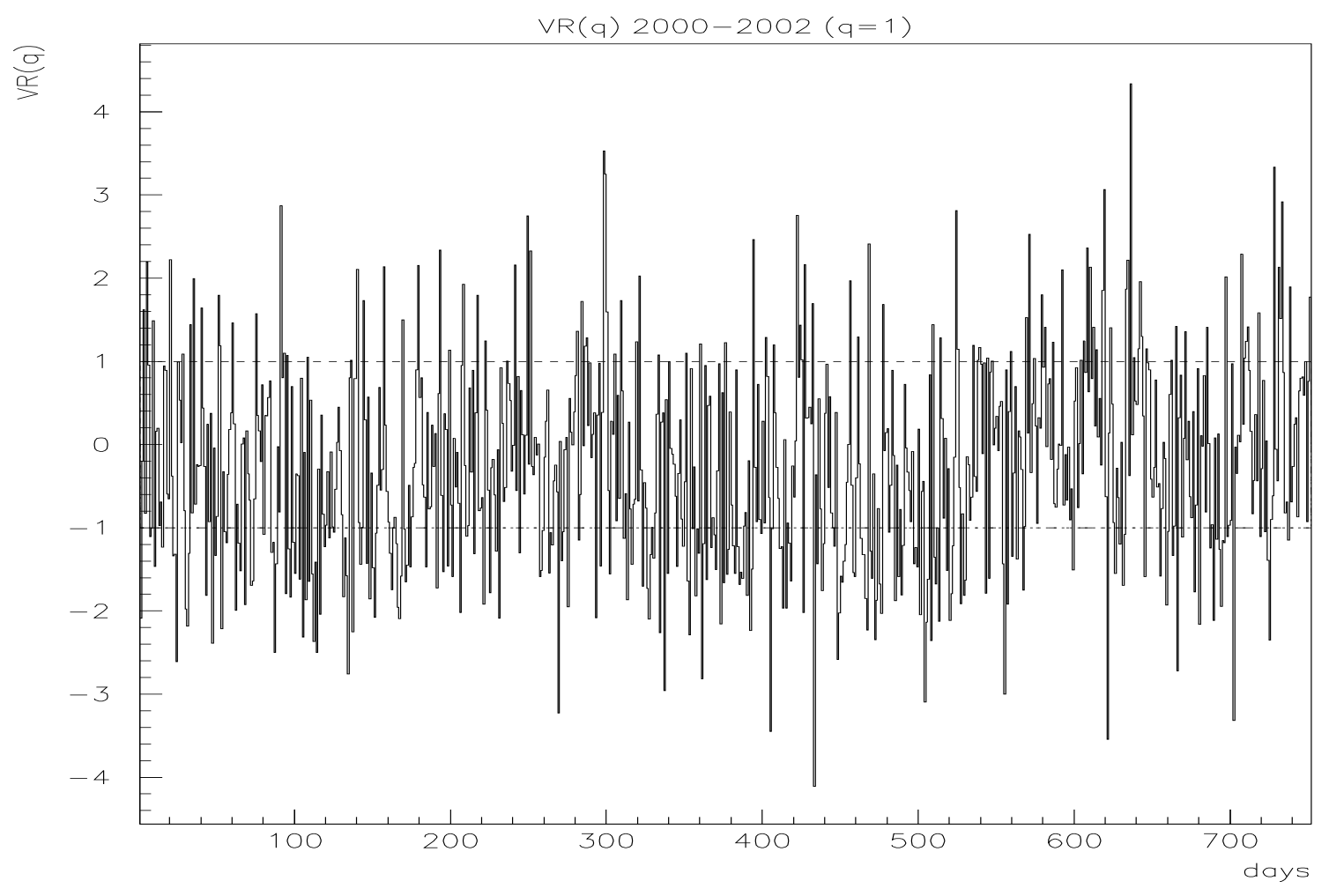

Figure 4: Time series of $\widetilde{V R(1)}$ as defined in equation (13).

- there is evidence of serial correlation in high-frequency prices of the Italian stock index futures;

- the serial correlation is mainly negative and it is non-negligible for periods smaller than 20 minutes;

- this observed negative serial correlation is due to the bid-ask bounce effect;

Up to now, then, nothing is very surprising. Similar conclusions have been drawn on other markets. Our results show that we cannot rule out the weak informational efficiency of the Italian market, even if we raise the frequency of observations.

In the next Section, we turn to the analysis of daily serial correlation with respect to other market variables. 


\begin{tabular}{|c|c|c|c|c|c|c|c|}
\hline$q$ & $\alpha$ & $\mathrm{t}$-test & $\widehat{V R}_{t-1}$ & $\mathrm{t}$-test & $V R_{t-2}$ & $\mathrm{t}$-test & $\mathrm{3}(5)$ \\
\hline \multirow{2}{*}{1} & -0.250 & -5.513 & 0.112 & $3.091^{* *}$ & & & $12.56^{*}$ \\
\cline { 2 - 8 } & -0.228 & -4.933 & 0.103 & $2.826^{* *}$ & 0.087 & $2.371^{* *}$ & 5.25 \\
\hline \multirow{2}{*}{10} & -0.349 & -8.653 & 0.111 & $3.061^{* *}$ & & & 4.08 \\
\cline { 2 - 8 } & -0.350 & -8.268 & 0.109 & $2.970^{* *}$ & 0.005 & 0.124 & 3.46 \\
\hline \multirow{2}{*}{20} & -0.391 & -10.548 & 0.026 & 0.712 & & & 4.97 \\
\cline { 2 - 8 } & -0.401 & -10.070 & 0.025 & 0.693 & -0.021 & -0.578 & 4.83 \\
\hline
\end{tabular}

Table 2: Estimates of the model (14) for different values of $q .{ }^{* *}$ denotes $99 \%$ significance, ${ }^{*}$ denotes $95 \%$ significance.

\section{Time series properties of intraday serial cor- relation}

In the previous Section we assessed the presence of serial correlation in the high frequency return time series. Here, we investigate the link between autocorrelations and some of the most important market variables. This can provide additional insight in the mechanism of price formation and in the trading process. First we analyze the presence of autoregressive patterns in the standardized variance ratio $\widetilde{V R}$ (for simplicity, we will omit the dependence from $q$ in what follows) time series, as suggested from the time series shown in Figure 4. We estimate the model:

$$
\widetilde{V R}_{t}=\alpha+\delta_{1} \cdot \widetilde{V R}_{t-1}+\delta_{2} \cdot \widetilde{V R}_{t-2}+\varepsilon_{t}
$$

where $\varepsilon_{t}$ is IID white noise, using OLS. We also estimate the restricted model with $\delta_{2}=0$. We test the specification of the model by the lag-5 Ljung-Box statistics on residuals. We report the results in Table 2 .

As we can see from the Table, there is evidence of first order autoregression of the $V R$ for at least $q=10$. If we consider $\widetilde{V R}(q=1)$ as a measure of serial correlations of returns time series, then it results that there are second order autoregressive patterns in serial correlations since, if the second-order autoregressive term is not included, the residuals are serially correlated and the model is misspecified. The $R^{2}$ of the proposed regressions are very low, ranging around $1 \%$.

These results could be an artifact of the pronounced heteroskedasticity of futures prices. Indeed, the VR measures are (standardized) ratios of 
realized volatilities, so they could be not serially independent. To check this, we estimate the regression (14) on a simulated time series, according to the GARCH model as in Section 2. We find that the significant autoregressive component is observed also in the simulated time series, thus it is a by-product of heteroskedasticity. In what follows, we then include the autoregressive component of standardized Variance Ratios in our regressions, and we check via the Ljung-Box test if we manage to remove the autocorrelation from the residuals.

\subsection{Serial correlation and volatility}

The impact of volatility on serial correlation patterns has already been discussed in the empirical literature. In particular, LeBaron (1992) found that, regarding daily and weekly data, there is evidence of a negative correlation between volatility and returns serial correlation, that is positive serial correlation is observed in low volatility regimes, and no serial correlation is observed in high volatility regimes. Similar results are found by Sentana and Wadhwani (1992). These results regard individual stocks and stock portfolios. This effect is sometimes regarded as LeBaron effect, see e.g. Dacorogna et al. (2001).

Now we focus our attention on intraday data, using realized measures of intraday serial correlation and intraday volatility. As before, we adopt as a measure of serial correlation of returns the quantity $\widetilde{V R}(q)$. Daily volatility is routinely evaluated using high-frequency data. To this purpose, we adopt a technique similar to realized volatility based on a trigonometric expansion, namely the Fourier method of Malliavin and Mancino (2002). The details on volatility computation on the same data set can be found in Renò and Rizza (2003). The time series of realized daily volatility is shown in Figure 5 . We then estimate via OLS the following model, for different values of the aggregation period $q$ :

$$
\widetilde{V R}_{t}=\alpha+\beta \cdot \log \left(\sigma_{t}^{2}\right)+\delta_{1} \cdot \widetilde{V R}_{t-1}+\delta_{2} \cdot \widetilde{V R}_{t-2}+\varepsilon_{t} .
$$

As before, we also estimate the restricted models $\delta_{2}=0$ and $\delta_{1}=\delta_{2}=0$. Results of the estimates are displayed in Table 3.

We find that there is strong correlation between serial correlations of intraday returns and daily volatility, for every considered value of the aggregation period $q$, and that the coefficient is positive. The relation is also apparent in the scatter plot of daily logarithmic volatility and standardized 


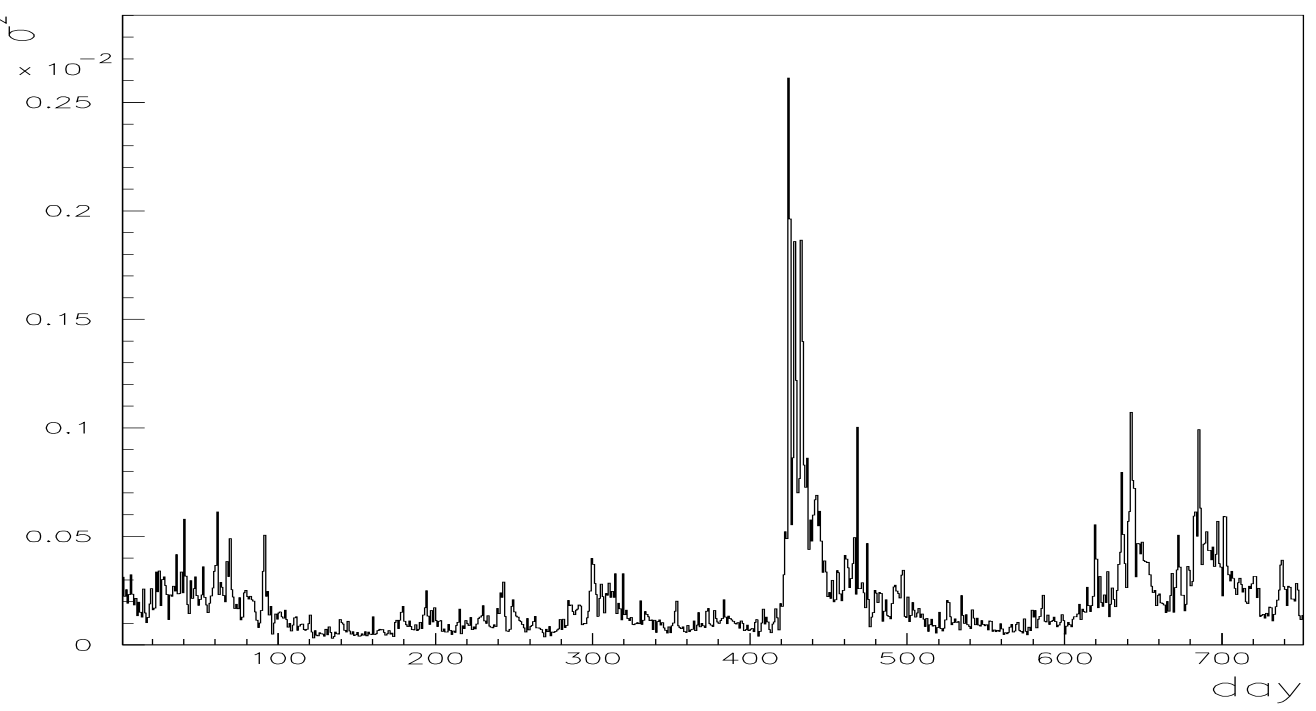

Figure 5: Time series of daily volatility. The largest peak corresponds to September 11, 2001

\begin{tabular}{|c|c|c|c|c|c|c|c|c|c|}
\hline$q$ & $\alpha$ & t-test & $\log \left(\sigma^{2}\right)$ & t-test & $V R_{t-1}$ & t-test & $\widetilde{V R} R_{t-2}$ & t-test & Q(5) \\
\hline \multirow{3}{*}{1} & 2.786 & 5.151 & 0.349 & $5.696^{* *}$ & & & & & $33.31^{* *}$ \\
\cline { 2 - 11 } & 2.648 & 4.860 & 0.330 & $5.336^{* *}$ & 0.081 & $2.231^{*}$ & & & $19.35^{* *}$ \\
\cline { 2 - 10 } & 2.557 & 4.679 & 0.318 & $5.113^{* *}$ & 0.075 & $2.051^{*}$ & 0.067 & 1.865 & 10.56 \\
\hline \multirow{3}{*}{10} & 2.434 & 5.291 & 0.321 & $6.161^{* *}$ & & & & & $18.28^{* *}$ \\
\cline { 2 - 10 } & 2.264 & 4.853 & 0.299 & $5.621^{* *}$ & 0.070 & 1.932 & & & 8.01 \\
\cline { 2 - 11 } & 2.287 & 4.873 & 0.303 & $5.640^{* *}$ & 0.070 & 1.930 & -0.024 & -0.650 & 8.93 \\
\hline \multirow{2}{*}{20} & 1.798 & 4.323 & 0.250 & $5.302^{* *}$ & & & & & 6.45 \\
\cline { 2 - 10 } & 1.798 & 4.266 & 0.250 & $5.214^{* *}$ & -0.010 & -0.270 & & & 6.52 \\
\cline { 2 - 10 } & 1.858 & 4.382 & 0.260 & $5.350^{* *}$ & -0.011 & -0.299 & -0.050 & -1.365 & 8.10 \\
\hline
\end{tabular}

Table 3: Results of the regression (15), for different values of $q$. Significance at 95\% is denoted by $a^{*}$, at $99 \%$ by $a^{* *}$.

VR, shown in Figure 6. This means that when volatility is high, the intraday serial correlation tends to be positive. When volatility is low, the intraday serial correlation tends to be negative. For $q$ less than 10 , the regression (15) turns out to be misspecified, according to the Ljung-Box statistics, unless the auto-regressive component of the standardized variance ratios is included. We remark that the positive link between standardized Variance 


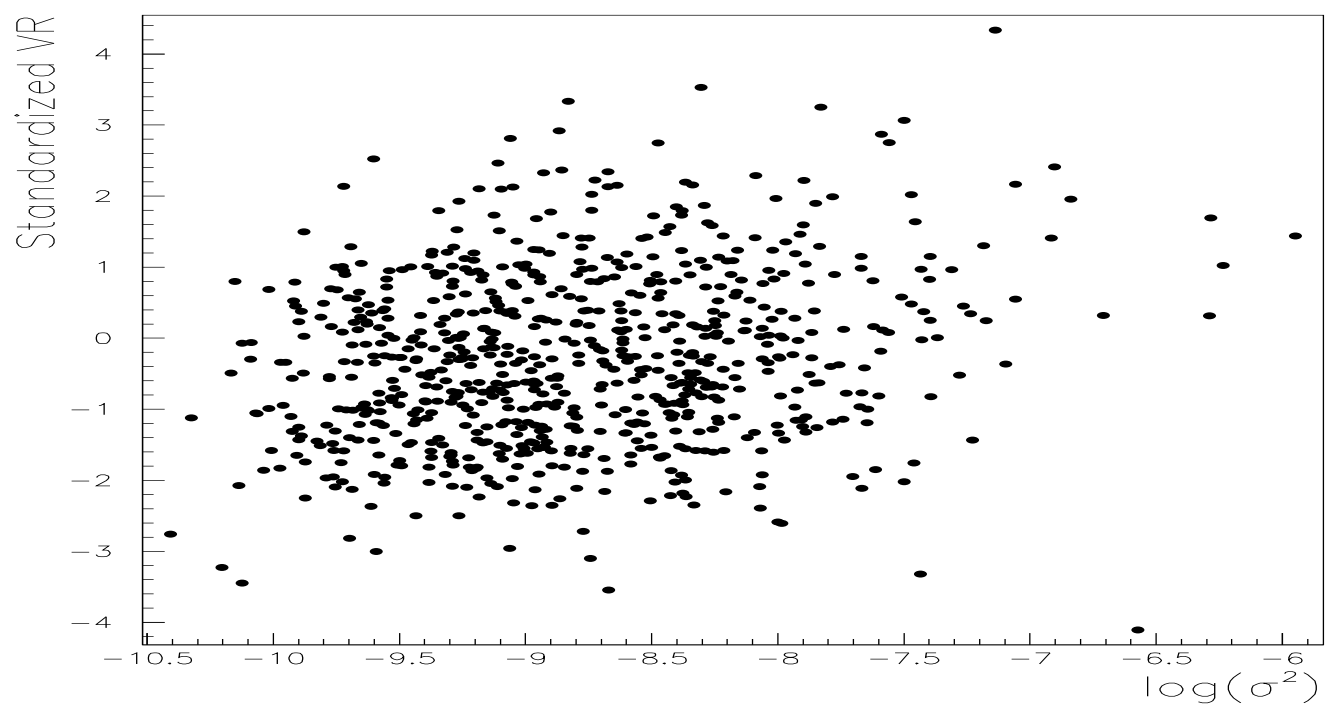

Figure 6: Scatter plot of daily logarithmic volatility versus standardized variance ratios

Ratios and volatility is not observed on simulated data.

Given the relevance of this result, we test it again with a different approach. We evaluate, as above, the percentage of violations of the null hypothesis of no serial correlation according to the $\widehat{V R(q)}$ statistics, considering several values of the aggregation period $q$, but now we consider only on those days of our data set in which volatility is larger than a given threshold. Table 4 reports these percentages. As we can see, when we select low volatility thresholds, serial correlations remain mainly negative. Instead, when we select high volatility days, positive serial correlations become more relevant and this behavior is confirmed for all the chosen values of the aggregation period $q$.

We remark that this finding is, in some sense, at odds with the findings of LeBaron (1992) and Sentana and Wadhwani (1992), who show that on daily and weekly data serial correlation of stocks and stock indexes declines with rising volatilities. At the intraday level, for the considered stock index future, the opposite holds. Our results are instead in agreement with the theoretical predictions of Chan (1993). According to his model, the adjustment of prices is conditioned by noisy signals about information and it is then reinforced by movements in other markets. The model is based on the idea of reinforcement of opinions. Since market makers cannot adapt 
$\sigma^{2}>10^{-4}, 68.7 \%$ of the sample

\begin{tabular}{lllllll}
\hline$q$ & $90 \%^{+}$ & $90 \%^{-}$ & $95 \%^{+}$ & $95 \%^{-}$ & $99 \%^{+}$ & $99 \%^{-}$ \\
\hline 1 & 0.124 & 0.203 & 0.081 & 0.101 & 0.029 & 0.023 \\
10 & 0.083 & 0.155 & 0.052 & 0.074 & 0.017 & 0.006 \\
20 & 0.060 & 0.147 & 0.039 & 0.048 & 0.006 & 0.002 \\
\hline \multicolumn{7}{c}{$\sigma^{2}>2 \cdot 10^{-4}, 34.2 \%$ of the sample } \\
\hline$q$ & $90 \%^{+}$ & $90 \%^{-}$ & $95 \%^{+}$ & $95 \%^{-}$ & $99 \%^{+}$ & $99 \%^{-}$ \\
\hline 1 & 0.148 & 0.167 & 0.093 & 0.097 & 0.031 & 0.023 \\
10 & 0.097 & 0.121 & 0.054 & 0.074 & 0.016 & 0.008 \\
20 & 0.062 & 0.140 & 0.039 & 0.039 & 0.004 & 0.004 \\
\hline \multicolumn{7}{c}{$\sigma^{2}>3 \cdot 10^{-4}, 16.4 \%$ of the sample } \\
\hline$q$ & $90 \%^{+}$ & $90 \%^{-}$ & $95 \%^{+}$ & $95 \%^{-}$ & $99 \%^{+}$ & $99 \%^{-}$ \\
\hline 1 & 0.195 & 0.146 & 0.122 & 0.098 & 0.049 & 0.041 \\
10 & 0.122 & 0.098 & 0.073 & 0.057 & 0.024 & 0.016 \\
20 & 0.073 & 0.114 & 0.041 & 0.049 & 0.000 & 0.008 \\
\hline$q$ & $\sigma^{2}>4 \cdot 10^{-4}, 8.3 \%$ of the sample \\
\hline 1 & $90 \%^{+}$ & $90 \%^{-}$ & $95 \%^{+}$ & $95 \%^{-}$ & $99 \%^{+}$ & $99 \%^{-}$ \\
10 & 0.226 & 0.129 & 0.161 & 0.113 & 0.081 & 0.032 \\
20 & 0.161 & 0.097 & 0.097 & 0.048 & 0.032 & 0.016 \\
\hline$q$ & 0.081 & 0.113 & 0.048 & 0.032 & 0.000 & 0.000 \\
\hline$q$ & $90 \%^{+}$ & $90 \%^{-}$ & $95 \%^{+}$ & $95 \%^{-}$ & $99 \%^{+}$ & $99 \%^{-}$ \\
\hline 1 & 0.310 & 0.119 & 0.214 & 0.095 & 0.119 & 0.048 \\
20 & 0.214 & 0.095 & 0.143 & 0.048 & 0.048 & 0.024 \\
\hline
\end{tabular}

Table 4: Reports the number of significant positive and negative VR, for different significance level, on subsamples of growing daily volatility. When volatility is high, the number of significant negative VRs is closer to the null, while the number of significant positive VRs increases.

their opinions instantaneously when they receive signals from similar assets, they react subsequently, and this mechanism induces correlation in pricing errors. One important consequence of this model is that serial correlation should be higher in days of high volatility. For the Italian stock index futures, there are plenty of more liquid substitutes, for example the EUROSTOXX futures, which can generate this effect.

Therefore, we can conclude that at intraday level there is no evidence 
of LeBaron effect, but the correlation between returns serial correlation and volatility has opposite sign with respect to what is observed at daily and weekly level.

The relation between intraday serial correlation and volatility is interesting, but it is well known that volatility is highly predictable in financial markets, than it could be more interesting to link serial correlation to the unexpected part of daily volatility. Daily realized volatility is known to be well described by an auto-regressive model (Andersen et al., 2003). We then estimate the following model:

$$
\log \left(\sigma_{t}^{2}\right)=\alpha+\beta_{1} \log \left(\sigma_{t-1}^{2}\right)+\beta_{2} \log \left(\sigma_{t-2}^{2}\right)+\varepsilon_{t} .
$$

Estimates are $\hat{\beta}_{1}=0.589, \hat{\beta}_{2}=0.126$, with an $R^{2}$ of 0.748 , which confirms the high predictability of daily volatility.

We then define the unexpected volatility as the residuals of the above regression, $\sigma_{u, t} \equiv \hat{\varepsilon}_{t}$. By construction, volatility and unexpected volatility are orthogonal, and we investigate whether there is a link between $V R(q)$ and unexpected volatility by estimating the following model:

$$
\widetilde{V R}_{t}=\alpha+\delta_{1} \cdot \widetilde{V R}_{t-1}+\delta_{2} \cdot \widetilde{V R}_{t-2}+\beta \cdot \sigma_{u, t}+\varepsilon_{t} .
$$

The results of this regression is shown in Table 5. As we can see, also the unexpected volatility influence is strong on variance ratios for all the considered $q$ values, Particularly, for $q=1$ and considering the second order autoregressive term in $V R(q)$ time series, we obtain a value for $Q(5)$ in agreement with the null hypothesis.

It is now interesting to study the influence of both volatility and unexpected volatility in the same regression. We do this by estimating the model:

$$
\widetilde{V R}_{t}=\alpha+\delta_{1} \cdot \widetilde{V R}_{t-1}+\delta_{2} \cdot \widetilde{V R}_{t-2}+\beta \cdot \log \left(\sigma_{t}^{2}\right)+\gamma \cdot \sigma_{u, t}+\varepsilon_{t} .
$$

Results are shown in Table 6. Our results indicate that, when regressing on both volatility and unexpected volatility, the latter is influential, while the first does not account for the observed serial correlation. Again, the autoregressive component of variance ratios is necessary for correct model specification. This result holds for every value of $q$, and more effectively for $q=1$.

It is important to stress that, as noted by Sentana and Wadhwani (1992), the increase in intraday serial correlation with larger volatility is 


\begin{tabular}{|c|c|c|c|c|c|c|c|c|c|}
\hline$q$ & $\alpha$ & t-test & $\sigma_{u}$ & t-test & $\widetilde{V R}_{t-1}$ & t-test & $\widetilde{V R}_{t-2}$ & t-test & Q(5) \\
\hline \multirow{4}{*}{1} & -0.285 & -6.821 & 1.165 & $9.937^{* *}$ & & & & & $26.89^{* *}$ \\
\cline { 2 - 11 } & -0.259 & -6.058 & 1.145 & $9.788^{* *}$ & 0.091 & $2.657^{* *}$ & & & $15.44^{* *}$ \\
\cline { 2 - 10 } & -0.234 & -5.383 & 1.154 & $9.909^{* *}$ & 0.080 & $2.329^{* *}$ & 0.099 & $2.883^{* *}$ & 7.61 \\
\hline \multirow{3}{*}{10} & -0.395 & -11.258 & 1.122 & $11.410^{* *}$ & & & & & $11.85^{*}$ \\
\cline { 2 - 10 } & -0.371 & -9.893 & 1.099 & $11.095^{* *}$ & 0.059 & 1.742 & & & 9.74 \\
\cline { 2 - 11 } & -0.368 & -9.355 & 1.099 & $11.089^{* *}$ & 0.058 & 1.704 & 0.008 & 0.240 & 9.49 \\
\hline \multirow{2}{*}{20} & -0.402 & -12.618 & 0.941 & $10.525^{* *}$ & & & & & $11.68^{*}$ \\
\cline { 2 - 10 } & -0.414 & -11.899 & 0.952 & $10.532^{* *}$ & -0.030 & -0.855 & & & 11.04 \\
\cline { 2 - 10 } & -0.425 & -11.390 & 0.954 & $10.543^{* *}$ & -0.029 & -0.836 & -0.028 & -0.812 & $11.82^{*}$ \\
\hline
\end{tabular}

Table 5: Results of the regression (17), for different values of $q$. Significance at $95 \%$ is denoted by $a^{*}$, at $99 \%$ by $a^{* *}$.

still compatible with equilibrium, since exploiting the anomaly is riskier with higher volatility.

\subsection{Serial correlation and trading volume}

Trading volume plays a key role in rational models describing the information flow, see e.g. Admati and Pfleiderer (1988). Campbell et al. (1993) develop a model in which serial correlation is due to changing risk-aversion. They argue that in days of high trading volume, serial correlation should be larger. Safvenvblad (2000) confirms empirically this prediction on Swedish stocks, but does not for the Swedish stock index. Since he finds that in his sample period high volume days are more often high return days, his explanation is profit taking. Conrad et al. (1994) find that high volume is associated with negative autocorrelation, while low volume is associated with positive autocorrelation. See also Chordia and Swaminathan (2000), who link the trading volume to the speed of adjustment of information, showing that high volume portfolios lead low volume portfolios.

We then investigate the relation among our intraday serial correlation measures $\widetilde{V R}(q)$ and daily volume, via the regression:

$$
\widetilde{V R}_{t}=\alpha+\delta_{1} \cdot \widetilde{V R}_{t-1}+\delta_{2} \cdot \widetilde{V R}_{t-2}+\beta \cdot V_{t}+\varepsilon_{t}
$$

where $V_{t}$ is the total volume transacted at day $t$. Table 7 shows the results.

We find that trading volume is significant in explaining the variability of variance ratios, and the relation is positive: in high trading volume days 
the intraday serial correlation is higher. However, it is important to stress that it is very difficult to disentangle the volume effect from the volatility effect, since these two quantities are strongly positively correlated. 


\begin{tabular}{|c|c|c|c|c|c|c|c|c|c|c|c|}
\hline$q$ & $\alpha$ & t-test & $\sigma_{t}^{2}$ & t-test & $\sigma_{u}$ & t-test & $\widehat{V R_{t-1}}$ & t-test & $\widehat{V R_{t-2}}$ & t-test & Q(5) \\
\hline \multirow[t]{3}{*}{1} & 0.390 & 0.651 & 0.077 & 1.129 & 1.088 & $8.032^{* *}$ & & & & & $27.14^{* *}$ \\
\hline & 0.181 & 0.301 & 0.050 & 0.732 & 1.096 & $8.115^{* *}$ & 0.087 & $2.511^{* *}$ & & & $15.73^{* *}$ \\
\hline & -0.027 & -0.045 & 0.024 & 0.342 & 1.131 & $8.376^{* *}$ & 0.078 & $2.257^{*}$ & 0.097 & $2.807^{* *}$ & 7.71 \\
\hline \multirow[t]{3}{*}{10} & 0.023 & 0.046 & 0.047 & 0.831 & 1.075 & $9.452^{* *}$ & & & & & $11.81^{*}$ \\
\hline & -0.083 & -0.163 & 0.033 & 0.570 & 1.067 & $9.392^{* *}$ & 0.056 & 1.632 & & & 9.67 \\
\hline & -0.093 & -0.182 & 0.031 & 0.538 & 1.069 & $9.363^{* *}$ & 0.056 & 1.612 & 0.005 & 0.149 & 9.50 \\
\hline \multirow[t]{3}{*}{20} & -0.271 & -0.591 & 0.015 & 0.288 & 0.926 & $8.955^{* *}$ & & & & & $11.55^{*}$ \\
\hline & -0.229 & -0.498 & 0.021 & 0.402 & 0.932 & $8.994^{* *}$ & -0.031 & -0.899 & & & 10.84 \\
\hline & -0.177 & -0.382 & 0.028 & 0.535 & 0.927 & $8.925^{* *}$ & -0.031 & -0.898 & -0.031 & -0.885 & 11.65 \\
\hline
\end{tabular}

Table 6: Results of regression (18), for different values of $q$. Significance at 95\% is denoted by $a^{*}$, at 99\% by $a^{* *}$.

\begin{tabular}{|c|c|c|c|c|c|c|c|c|c|}
\hline$q$ & $\alpha$ & t-test & $V_{t}$ & t-test & $V R_{t-1}$ & t-test & $V R_{t-2}$ & t-test & Q(5) \\
\hline \multirow{2}{*}{1} & -1.524 & -10.150 & $0.726 \mathrm{E}-04$ & $8.604^{* *}$ & & & & & $29.29^{* *}$ \\
\cline { 2 - 10 } & -1.485 & -9.695 & $0.716 \mathrm{E}-04$ & $8.407^{* *}$ & 0.068 & 1.940 & & & $19.98^{* *}$ \\
\cline { 2 - 10 } & -1.450 & -9.395 & $0.706 \mathrm{E}-04$ & $8.265^{* *}$ & 0.061 & 1.725 & 0.067 & 1.902 & 11.00 \\
\hline \multirow{2}{*}{10} & -1.716 & -13.785 & $0.777 \mathrm{E}-04$ & $11.097^{* *}$ & & & & & $16.59^{* *}$ \\
\cline { 2 - 10 } & -1.668 & -12.844 & $0.758 \mathrm{E}-04$ & $10.613^{* *}$ & 0.044 & 1.280 & & & 10.53 \\
\cline { 2 - 10 } & -1.686 & -12.762 & $0.761 \mathrm{E}-04$ & $10.598^{* *}$ & 0.046 & 1.324 & -0.031 & -0.895 & $13.13^{*}$ \\
\hline \multirow{2}{*}{20} & -1.487 & -13.122 & $0.637 \mathrm{E}-04$ & $10.000^{* *}$ & & & & & 7.49 \\
\cline { 2 - 10 } & -1.511 & -12.799 & $0.643 \mathrm{E}-04$ & $9.929^{* *}$ & -0.030 & -0.851 & & & 9.25 \\
\cline { 2 - 10 } & -1.547 & -12.843 & $0.652 \mathrm{E}-04$ & $10.011^{* *}$ & -0.029 & -0.847 & -0.054 & -1.551 & $13.39^{*}$ \\
\hline
\end{tabular}

Table 7: Results of the regression (19), for different values of $q$. Significance at $95 \%$ is denoted by $a^{*}$, at $99 \%$ by $a^{* *}$. 


\section{Conclusions}

In this paper, we study the serial correlation of intraday returns of the Italian stock index futures, the FIB30. Our first aim is to assess the efficiency of the Italian market. To this purpose, we adopt the Variance Ratio test, which needs to be carefully implemented on high-frequency prices. Our preliminary analysis show that interpolating the data to obtain one-minute returns allows to prevent microstructure effects from distorting the variance estimates. We also show, via Monte Carlo simulations, that with oneminute returns following a $\operatorname{GARCH}(1,1)$ model, the small sample distribution of Variance Ratio is very close to the asymptotic distribution, under the null hypothesis, when the aggregation period is less than twenty minutes.

Our results show that the serial correlation is mostly negative, but this effect can be completely explained by the bid-ask bounce effects. Thus, by looking at variance ratio only, we support the efficiency of the Italian futures market.

However, our estimating technique provides us with a time series of variance ratio (more precisely, of standardized variance ratios), of which we can study the dynamic properties. Motivated by earlier literature, we study the connection of serial correlation, as quantified by the standardized VR, with daily volatility. We can easily assess this task, since we have both realized measures of serial correlations (the standardized variance ratios) and realized measures of daily volatilities, obtained with high-frequency data. We find that at the intraday level we have a different behavior from what is observed at daily and weekly level: when volatility is high, intraday serial correlation becomes positive. This effect can be explained in the framework of the Chan (1993) model, according to which traders react only partially to information, and then reinforce their opinions when they observe the movements of liquid substitutes. We also show that it is mainly the unexpected part of volatility to account for the evolution of serial correlation: the predictable part looks like not affecting the traders' behavior. We finally find that the serial correlation is positively linked to trading volume.

While this study is, to our knowledge, the first one to provide dynamical insights in the intraday serial correlation dynamics, we think that additional work is needed. In particular, it would be interesting to check whether our results are valid in a more liquid futures market, like the SPX500, or for individual stocks. It is clear that going below the daily level can help in understanding the trading mechanism. In this paper, we show that these 
analysis can be carried out with the classical VR statistics, with some adjustments. We think this can be an interesting topic for future research.

\section{References}

Admati, A. and P. Pfleiderer (1988). A theory of intraday patterns: Volume and price variability. The Review of Financial Studies 1(1), 3-40.

Ahn, D.-H., J. Boudoukh, M. Richardson, and R. Whitelaw (2002). Partial adjustment or stale prices? Implications from stock index and futures return autocorrelations. Review of Financial Studies 15, 655-689.

Andersen, T. and T. Bollerslev (1997). Intraday periodicity and volatility persistence in financial markets. Journal of Empirical Finance 4, 115158.

Andersen, T., T. Bollerslev, and A. Das (2001). Variance-ratio statistics and high-frequency data: Testing for changes in intraday volatility patterns. Journal of Finance 56(1), 305-327.

Andersen, T., T. Bollerslev, F. Diebold, and P. Labys (2000). Great realizations. Risk 13, 105-108.

Andersen, T., T. Bollerslev, F. Diebold, and P. Labys (2003). Modeling and forecasting realized volatility. Econometrica 71, 579-625.

Andersen, T., T. Bollerslev, and S. Lange (1999). Forecasting financial market volatility: Sample frequency vis-à-vis forecast horizon. Journal of Empirical Finance 6, 457-477.

Badrinath, S. G., J. R. Kale, and T. H. Noe (1995). Of shepherds, sheep, and the cross-autocorrelation in equity returns. Review of Financial Studies 8 , 401-430.

Barndorff-Nielsen, O. E. and N. Shephard (2002). Econometric analysis of realised volatility and its use in estimating stochastic volatility models. Journal of the Royal Statistical Society, Series B 64, 253-280.

Barucci, E. and R. Renò (2002a). On measuring volatility and the GARCH forecasting performance. Journal of International Financial Markets, Institutions and Money 12, 183-200. 
Barucci, E. and R. Renò (2002b). On measuring volatility of diffusion processes with high frequency data. Economics Letters 74, 371-378.

Bollerslev, T. and I. Domowitz (1993). Trading patterns and prices in the interbank foreign exchange market. Journal of Finance 48, 1421-1443.

Bollerslev, T., R. Engle, and D. Nelson (1994). Arch models. In R. Engle and D. McFadden (Eds.), Handbook of Econometrics, Volume IV. Amsterdam: North-Holland.

Boudoukh, J., M. Richardson, and R. Whitelaw (1994). A tale of three schools: insights on autocorrelations of short-horizon stock. Review of financial studies 7(3), 539-573.

Campbell, J., S. Grossman, and J. Wang (1993). Trading volume and serial correlation in stock returns. Quarterly journal of economics 108(4), 905939.

Cecchetti, S. G. and P. Sang Lam (1994). Variance-ratio tests: small-sample properties with an application to international output data. Journal of Business Economics and Statistics 12(2), 177-186.

Chan, K. (1992). A further analysis of the lead-lag relationships between the cash market and the stock index futures market. Review of financial studies $5,123-152$.

Chan, K. (1993). Imperfect information and cross-autocorrelation among stock prices. Journal of Finance 48(4), 1211-1230.

Chordia, T. and B. Swaminathan (2000). Trading volume and crossautocorrelations in stock returns. Journal of Finance 55(2), 913-935.

Conrad, J., A. Hameed, and C. Niden (1994). Volume and autocovariances in short-horizon individual security returns. Journal of Finance 49(4), 1305-1329.

Conrad, J. and G. Kaul (1988). Time variation in expected returns. Journal of Business 61, 489-519.

Conrad, J. and G. Kaul (1989). Mean reversion in short-horizon expected returns. Review of financial studies 2(2), 225-240. 
Corsi, F., G. Zumbach, U. Muller, and M. Dacorogna (2001). Consistent high-precision volatility from high-frequency data. Economic Notes 30(2), 183-204.

Cutler, D., J. Poterba, and L. Summers (1991). Speculative dynamics. Review of economic studies 58, 529-546.

Dacorogna, M., R. Gencay, U. Muller, R. Olsen, and O. Pictet (2001). An introduction to high-frequency finance. Academic Press.

Dacorogna, M., U. Muller, R. J. Nagler, R. Olsen, and O. Pictet (1993). A geographical model for the daily and weekly seasonal volatility in the FX market. Journal of International Money and Finance 12, 413-438.

Deo, R. S. and M. Richardson (2003). On the asymptotic power of the variance ratio test. Econometric Theory 19.

Drost, F. and T. Nijman (1993). Temporal aggregation of GARCH processes. Econometrica 61(4), 909-927.

Fama, E. (1970). Efficient capital markets: a review of theory and empirical work. Journal of Finance 25, 383-417.

Faust, J. (1992). When are variance ratio tests for serial dependence optimal? Econometrica 60(5), 1215-1226.

Goodhart, C. and M. O'Hara (1997). High frequency data in financial markets: Issues and applications. Journal of Empirical Finance 4, 73-114.

Ito, T., R. Lyons, and M. Melvin (1998). Is there private information in the FX market? The Tokio experiment. Journal of Finance 53, 1111-1130.

Jegadeesh, N. and S. Titman (1993). Returns on buying winners and selling losers: implications for market efficiency. Journal of Finance 48, 65-91.

Kanatani, T. (2004). Integrated volatility measuring from unevenly sampled observations. Economics Bulletin 3(36), 1-8.

Kim, M. J., C. R. Nelson, and R. Startz (1991). Mean reversion in stock prices? a reappraisal of the empirical evidence. Review of Economic Studies 58, 515-528.

LeBaron, B. (1992). Some relations between volatility and serial correlations in stock market returns. Journal of Business 65(2), 199-219. 
Llorente, G., G. Michaely, G. Saar, and J. Wang (2002). Dynamic volumereturn relation of individual stocks. Review of Financial Studies 15, 10051047.

Lo, A. W. and A. C. MacKinlay (1988). Stock market prices do not follow random walks: evidence from a simple specification test. Review of financial studies 1, 41-66.

Lo, A. W. and A. C. MacKinlay (1989). The size and the power of the variance ratio test in finite samples: a Monte Carlo investigation. Journal of Econometrics 40, 203-238.

Lo, A. W. and A. C. MacKinlay (1990a). An econometric analysis of nonsynchronous trading. Journal of Econometrics 45, 181-211.

Lo, A. W. and A. C. MacKinlay (1990b). When are contrarian profits due to stock market overreaction? Review of financial studies 3, 175-205.

Low, A. and J. Muthuswamy (1996). Information flows in high frequency exchange rates. In C. Dunis (Ed.), Forecasting Financial Markets: Exchange Rates, Interest rates and Asset Management, pp. 3-32. John Wiley \& Sons.

Malliavin, P. and M. Mancino (2002). Fourier series method for measurement of multivariate volatilities. Finance \& Stochastics 6(1), 49-61.

Mech, T. (1993). Portfolio return autocorrelation. Journal of Financial Economics 34, 307-344.

Pan, M.-S., K. Chan, and R. Fok (1997). Do currency futures prices follow random walk? Journal of Empirical Finance 4, 1-15.

Patro, D. K. and Y. Wu (2004). Predictability of short-horizon returns in international equity markets. Journal of Empirical Finance 11, 553-584.

Poterba, J. and L. Summers (1988). Mean reversion in stock returns: evidence and implications. Journal of Financial Economics 22, 27-60.

Renò, R. and R. Rizza (2003). Is volatility lognormal? Evidence from Italian futures. Physica A 322, 620-628.

Richardson, M. and T. Smith (1993). Test of financial models in the presence of overlapping observations. Review of Financial Studies 4(2), 227-254. 
Roll, R. (1984). A simple measure of the implicit bid-ask spread in an efficient market. Journal of Finance 39, 1127-1139.

Safvenvblad (2000). Trading volume and autocorrelation: empirical evidence from the Stockholm Stock Exchange. Journal of Banking and Finance 24(8), 1275-1287.

Sentana, E. and S. Wadhwani (1992). Feedback traders and stock return autocorrelation: evidence from a century of daily data. Economic Journal 102, 415-425.

Thomas, S. and T. Patnaik (2003). Variance-ratio tests and high-frequency data: a study of liquidity and mean reversion in the indian equity markets. Working Paper. 